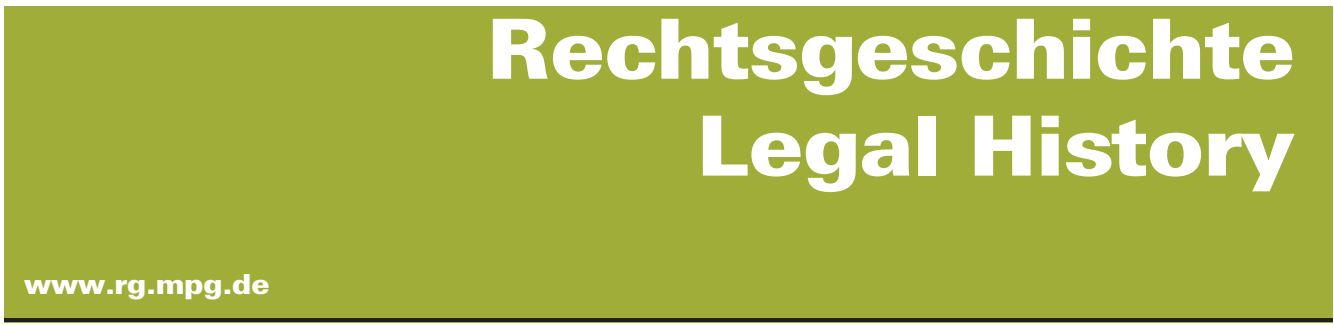

http://www.rg-rechtsgeschichte.de/rg21

Rg 212013 189-191

Zitiervorschlag: Rechtsgeschichte - Legal History Rg 21 (2013)

http://dx.doi.org/10.12946/rg21/189-191

\title{
Diego Quaglioni
}

\section{«The Outer and the Inner Aspects of Social Life»}




\section{Diego Quaglioni «The Outer and the Inner Aspects of Social Life»}

Dieci anni fa, nel 2003, a venti anni dalla pubblicazione di Law and Revolution. The Formation of the Western Legal Tradition, Harold Berman volle intitolare il suo ultimo libro, ancora una volta e puntigliosamente, Law and Revolution. Nel sottotitolo, The Impact of the Protestant Reformations on the Western Legal Tradition, il nuovo volume sottolineava insieme l'unità della tradizione giuridica occidentale e ciò che in altra occasione Berman aveva chiamato «the radical jurisprudential break brought about by the Lutheran revolution against the Roman Catholic Church». ${ }^{1}$

Continuità e discontinuità: l'ultima riflessione di Berman riproponeva i tratti della "struttura basilare" della tradizione giuridica occidentale, col suo dualismo tra spirituale e secolare, tra norma morale-religiosa e norma giuridica, tra coscienza e legge. Il secondo volume interpretava il primo, ${ }^{2}$ ancora una volta revocando in dubbio, in modo radicale e volutamente unilaterale, intiere categorie storiografiche e consolidate cronologie, nonché l'uso di schemi, come quelli correnti nella comparatistica di tradizione anglo-americana, tesi a negare l'unità della tradizione giuridica occidentale separando in modo del tutto antistorico $\mathrm{i}$ "grandi sistemi" di civil law da quelli di common law. ${ }^{3}$

Oggi, a trent'anni dal primo Law and Revolution e a dieci dal secondo, il lascito fondamentale di Berman alla storiografia giuridica occidentale e alla storia giuridica comparata può essere colto in un richiamo, allo stesso tempo scientifico e morale, a rivedere le linee convenzionali lungo le quali tanta parte della letteratura storico-giuridica del nostro tempo si è adagiata, compiacendosi talvolta di enfatizzare "tipicità" epocali ed epocali fratture e perdendo di vista il problema storico-attuale della tradizione giuridica occidentale e della sua unità. ${ }^{4}$ Di tale unità Berman ha mostrato la natura internamente dialettica, articolata e intessuta di "Grandi Rivoluzioni”, cioè la paradossale continuità di un carattere incessantemente discontinuo, e dunque il carattere evolutivo, progressivo di una tradizione capace di una continua rielaborazione nella conservazione della sua struttura basilare, almeno fino al dissolversi del comune patrimonio giuridico dell'Occidente nelle violenze dei nazionalismi del XX secolo. Si tratta di una concezione che risale a Out of Revolution di Eugen Rosenstock-Huessy (1938), e che in Berman assume il sembiante di un pensiero storico-giuridico (historical jurispruden$c e$ ) in cui la storia svolge un ruolo risolutore delle tensioni tra etica e politica. La storia giuridica assume in Berman il ruolo di una integrative jurisprudence, ${ }^{5}$ superando l'unilateralità sia del positivismo giuridico sia delle istanze neo-giusnaturalistiche e recuperando il senso della tradizione giuridica nel vincolo con i suoi belief systems. ${ }^{6}$

I venti anni che corrono dal primo Diritto $e$ rivoluzione, il libro che Richard Helmholz e Reinhard Zimmermann hanno giudicato come uno dei massimi contributi della storiografia giuridica del nostro tempo, ${ }^{7}$ sono anche, per Berman, lo spazio dell'ulteriore declino dell'Occidente e del progressivo venir meno della coscienza della sua tradizione giuridica. È da questo punto di vista che Berman volge lo sguardo alle conseguenze, sul piano della storia costituzionale d'Europa (e guardando agli Stati Uniti d'America), delle trasformazioni di quella stessa tradizione e delle sue basi etico-religiose nel grande sommovimento dei sistemi di valori della cristianità nelle Riforme luterana e calvinista dei secoli XVI e XVII. Berman avverte che tale "impatto" deve considerarsi «come parte di una storia ancora più ampia, in una "prospettiva millenaria”, cioè come parte della tradizione giuridica occidentale e dei suoi istituti caratteristici, tanto nel diritto pubblico quanto in quello privato.

La prospettiva interpretativa indicata dallo storico americano esprime una linea di pensiero il cui significato non è limitato, come talvolta si è voluto credere, alla "scoperta" del carattere dirompente dell'istituzionalizzarsi, nei secoli XI-XII, di un dualismo permanente e di una tensione concorrenziale tra due poli, lo spirituale e il temporale,
1 Berman/Reid (1992) 497f.

2 Quaglioni (2010) XII.

3 Berman (2003).

4 Birocchi/Quaglioni / Mazzacane (2011).
5 Hunter (ed.) (1996).

6 Berman (2003) 497.

7 Helmholz (1993); ZimmermanN (2008). 
ognuno dei quali tende ad assumere una posizione egemonica nella società e nella tradizione giuridica occidentale. Essa riguarda invece, ancora una volta, la sua "crisi" presente, dalla quale è possibile osservare gli snodi traumatici, i sommovimenti spirituali dai quali ha origine, lungo i secoli XVI e XVII, una nuova dimensione del potere e una nuova stagione del diritto e del pensiero giuridico: la "prima modernità" e la "secolarizzazione" secondo la storiografia convenzionale; la "seconda modernità" e la "spiritualizzazione del secolare" secondo Berman. ${ }^{8}$

Nella lunga tradizione giuridica occidentale quel radical breack non separa il "medievale" dal "moderno", ma divide in due la modernità stessa. Nulla che si avvicini al vecchio e anacronistico vizio dei "precorrimenti" e delle "anticipazioni" di concetti e istituti giuridici, nulla che sappia di antistorica proiezione all'indietro di categorie ed esperienze della "modernità". La storia delle rivoluzioni e del loro "impatto" sulla tradizione giuridica occidentale si svolge in un continuo intreccio con le principali correnti di pensiero e della storiografia del secolo scorso, in una stretta dialettica con la prospettiva interpretativa marxista e weberiana, che Berman respinge in nome di una concezione "integrale" del diritto come fatto spirituale e sociale, fino a smentire i giudizi recenti sul carattere "anti-romanistico" della tradizione del diritto inglese (che per Berman è storicamente e indissolubilmente parte della storia del ius commune e delle sue trasformazioni nella modernità, al di là di ogni mitologia giuridica ottocentesca) o a segnalare la debolezza di esercizi d'interpretazione della tradizione giusdottrinale in chiave sociologica.

Law and Revolution resta un documento tra i più interessanti della storiografia giuridica del nostro tempo, espressione di una proposta interpretativa ineludibile, se non altro perché essa si presenta esattamente come il rovesciamento dell'ormai stantia fabula dell'origine dei principali concetti del diritto pubblico europeo dalla "secolarizzazione" di concetti teologici. Penso ad una tempestiva e densa riflessione di Paolo Prodi, a cui si deve la recezione in Italia del primo Diritto e rivoluzione e che in alcune delle sue opere maggiori ha in seguito costantemente e criticamente dialogato con Berman e con la sua visione della crisi del diritto in Occidente. ${ }^{9}$

L'opera di Berman è semplicemente insostituibile come espressione al massimo livello di un moto di affermazione della storicità del diritto e dell'esigenza di riscoprire la relazione storicoattuale tra il diritto e i suoi belief systems, ravvivatasi nella letteratura storica più recente. ${ }^{10} \mathrm{Ne}$ è testimone, nel 1993, a mezzo del cammino fra il primo e il secondo Law and Revolution, la raccolta di saggi intitolata Faith and Order. The Reconciliation of Law and Religion. Vi sono raccolti tutti i maggiori temi della storiografia bermaniana, sintetizzati in un'importante introduzione dedicata alla dimensione religiosa del diritto. Alcuni dei saggi che vi si trovano raccolti costituiscono sviluppi del primo Law and Revolution e anticipazioni del secondo, come Berman stesso riconosce nella breve prefazione alla raccolta, avvertendo il lettore contro ogni indebita semplificazione della relazione tra diritto e religione: ${ }^{\mathbf{1 1}}$

"There are, to be sure, some people in the academic world, and many in what may be called the real world, who connect law with religion by emphasizing the link which both have with morality. On the theoretical level, it is argued that law is based ultimately on certain moral principles (contracts should be kept, crimes should be punished, civil rights should be protected and civil wrongs should be remedied, trials should be fair, and, in general, justice should be done), and that morality, in turn, including legal morality, is based ultimately on religion. A related, though different, argument is made by some religious moralists, namely, that society has a duty to enact laws prohibiting conduct which violates their religious morality. I do not, however, address these themes, except indirectly, and must therefore warn those who see in morality the only connection, or the basic

8 Dilcher (2009).

9 Prodi (1988); Prodi (1992); Prodi (1993); Prodi (2000).

10 STrohm (2008); STROHM/DE Wall (2009); cf. Stolleis (2009); Bianchin (2009).

11 Berman (1993) x. 
connection, between law and religion that they may be disappointed by this book. It is not only, and not primarily, about legal and religious morality. It is primarily about what I have called the religious dimension of law and the legal dimension of religion. It is about the dynamics of the interaction between these two great forces, which may be said to constitute the outer and the inner aspects of social life».
«The outer and the inner aspects of social life». Questa sembra a me la chiave principale per una lettura del lascito dell'opera storiografica di Harold Berman, ed anche la ragione per cui lo stesso Berman, parafrasando Maitland, amava dire che la storia del diritto in Occidente non è stata ancora scritta. ${ }^{12}$

\section{Bibliografia}

- Berman, Harold J. (1984), Why the History of Western Law is not Written, in: University of Illinois Law Review, 511-520

- Berman, Harold J. (1993), Law and Order. The Reconciliation of Law and Religion, Grand Rapids (Michigan), Cambridge

- Berman, Harold J. (2003), Law and Revolution, 2. The Impact of the Protestant Reformations on the Western Legal Tradition, Cambridge (Mass.) and London

- Berman, Harold J., Reid, Charles J. Jr. (1993), Recensione di Kelly, John M. (1992), A Short History of Western Legal Theory, New York, in: The American Journal of Legal History 37, 497-499

- Bianchin, Lucia (2009), Recensione di Strohm, Christoph (2008), Calvinismus und Recht. Weltanschaulich-konfessionelle Aspekte im Werk reformierter Juristen in der Frühen Neuzeit, Tübingen, in: Sehepunkte 9

- Birocchi, Italo, Diego Quaglioni, Aldo Mazzacane (2011), La tradizione giuridica occidentale nella prospettiva della sua crisi presente, in: Quaderni fiorentini per la storia del pensiero giuridico moderno 40, 1031-1059

- Dilcher, Gerhard (2009), Säkularisierung von Herrschaft durch Sakralisierung der Gerechtigkeit?, in: Kroppenberg, Inge, Martin Löhnig, Dieter Schwab, Recht - Religion - Verfassung. Festschrift für Hans-Jürgen Becker zum 70. Geburtstag, Bielefeld, 9-47

- Helmholz, Richard (1993), Harold Berman's Accomplishment as a Legal Historian, in: Emory Law Journal 42, 475-496

- Hunter, Howard O. (ed.) (1996), The Integrative Jurisprudence of Harold J. Berman, Boulder (Col.), Oxford

- Prodi, Paolo (1988), Suggestioni (da H.J. Berman) per lo studio del ruolo del diritto papale tra medio evo ed età moderna, in: Nuovi moti per la formazione del diritto, Padova, 93-103

- Prodi, Paolo (1992), Il sacramento del potere. Il giuramento politico nella storia costituzionale dell'Occidente, Bologna

- Prodi, Paolo (Hg.) (1993), Glaube und Eid. Treueformeln, Glaubensbekenntnisse und Sozialdisziplinierung zwischen Mittelalter und Neuzeit, München

- Prodi, Paolo (2000), Una storia della giustizia. Dal pluralismo dei fori al moderno dualismo tra coscienza e diritto, Bologna

- Quaglioni, Diego (2010), Presentazione dell'edizione italiana, in: Berman, Harold J., Diritto e rivoluzione, II. L'impatto delle riforme protestanti sulla tradizione giuridica occidentale. Edizione italiana, Quaglioni, Diego (ed.), Bologna

- Stolleis, Michael (2009), Genf, Heidelberg, Emden, in: Rechtsgeschichte 14, 196-199

- Sтroнm, Снгіsторн (2008), Calvinismus und Recht. Weltanschaulich-konfessionelle Aspekte im Werk reformierter Juristen in der Frühen Neuzeit, Tübingen

- Strohm, Christoph, Heinrich de Wall (Hg.) (2009), Konfessionalität und Jurisprudenz in der frühen Neuzeit, Berlin

- Zimmermann, Reinhard (2008), Harold Berman: Some Personal Reflections, in: Emory Law Journal 57, 1465-1469 